\title{
Ovarian Failure
}

National Cancer Institute

\section{Source}

National Cancer Institute. Ovarian Failure. NCI Thesaurus. Code C113351.

The inability of the ovaries to function. 